\title{
Extremum Seeking Control of Distributed Energy Resources with Decaying Dither and Equilibrium-based Switching
}

\author{
Michael D. Sankur \\ Lawrence Berkeley Lab \\ msankur@lbl.gov
}

\author{
Daniel B. Arnold \\ Lawrence Berkeley Lab \\ dbarnold@lbl.gov
}

\begin{abstract}
Optimal control of Distributed Energy Resources (DER) may be a critical component for proper operation of the electric distribution grid in the near future. However, many optimization-based approaches for managing DER require knowledge of the underlying distribution system topology, network impedances, and access to feeder-wide real time load information. In order to ameliorate these requirements, we propose a 2-dimensional Extremum Seeking (2D-ES) control scheme to manage DER active and reactive power contributions. We augment the 2D-ES scheme with an exponentially decaying probing (dither) signal that activates based on an equilibrium-based switching criteria. Our simulation results show that the approach can enable substation real and reactive power target tracking with dither signals that exponentially decay once the individual ES controllers have each reached their optimum values.
\end{abstract}

\section{Introduction}

Control of Distributed Energy Resources (DER) are a critical component of the Smart Grid. As DER continue to proliferate, it is expected that these devices will contribute significantly to maintaining the efficient and reliable operation of the electric power distribution system. Already, there is concern that the present level of DER deployed in the distribution grid will adversely affect grid reliability and resiliency. These worries will undoubtedly increase as the number of DER (particularly solar photovoltaic systems) grow over the next several decades. In places like California, which recently mandated solar $\mathrm{PV}$ systems be installed on all new builds beginning in 2020 [1], it will behoove grid operators to utilize DER to support the safe and efficient transfer of electricity in their systems.

In literature, there are no shortage of strategies that consider how DER should be managed to realize this vision. Practitioners of mathematical optimization often cast control of DER in distribution grids as a mathematical program (popularly referred to as Optimal Power Flow, or OPF). In [2], the authors formulate the decision-making process as a Semi-Definite Program (SDP). The work of [3] discusses convex relaxations for OPFs. The authors of [4] extend OPF formulations to handle uncertainties via the incorporation of chance constraints into the optimization formulation. In [5] the authors consider the use of the Alternating Direction Method of Multipliers (ADMM) to distribute the optimization problem. A recent work of the authors [6] discusses a linearized unbalanced power flow model and application to OPFs.

While OPF formulations are attractive as they can achieve very high levels of performance in terms of solution optimality, these methods typically rely on knowledge of the system model (topology and line segment impedances) and access to global load consumption information. Additionally, the success of these techniques is predicated on the existence of a communications infrastructure to relay sensing and actuation signals between DER and (possibly) a centralized decision-maker. The up-front cost and upkeep of such an infrastructure may prohibit such techniques from being realized in practice.

To address these issues, the authors have studied the application of Extremum Seeking (ES) to manage DER. ES is a nonlinear control technique that has become increasingly popular due to the ability of the approach to perform optimization in real time, without any knowledge of the system over which it is optimizing [7], [8]. The scheme is robust to plants with dynamics provided those dynamics act on a slower timescale than that of the ES control. In a previous work [9], we utilized two dimensional Extremum Seeking control to simultaneously manage the active and reactive power output of a controllable DER (where a separate ES loop is used to mange each power channel). The strategy alleviates the need for exogenous information such as network models, global knowledge of loads, and a large communications infrastructure. The approach 
has been shown to be robust to disturbances caused by intermittent renewable generation, changing loads, and the switching of legacy voltage regulation equipment. Obviously, the ability to control the real power output of PV arrays or battery storage systems is dependent on the availability of sufficient solar insolation or battery state of charge.

In the scheme, individual DER, each controlled by a separate 2D-ES controller, modulate their active and reactive power consumptions/injections sinusoidally. These sinusoids propagate through the network, affecting system voltages and power flows. Measurements from locations in the network where DER are to be used regulate system states are then collected. From these measurements, a system-wide fitness function (essentially an OPF objective function) is computed and broadcast to all DER. Using this broadcast objective, which is a scalar value, the ES controllers can extract their respective gradients needed to perform optimization. Obviously, the speed of the approach is dependant on the rate at which data can be collected from points of interest in the network and the speed of objective function computation and broadcast. The overall scheme presents less of a burden from an implementation standpoint as it only requires measurements at points of the network where control action is desired (unlike centralized OPF approaches that require knowledge of all system loads). The ES approach has been successfully demonstrated in a power hardware in the loop environment in controlling real PV inverters for feeder voltage regulation [10].

As our past work has been aimed demonstrating the technical potential of ES to control DER, we have not evaluated the economic cost/benefit for individual asset owners were they to be incorporated into this control paradigm. It is true that present compensation schemes do not incentivize resources to participate in this activity. However, in demonstrating the ability of ES-managed DER to enable feeder-level voltage and power control it is possible that other compensation schemes could be created to facilitate this solution.

A main drawback of the ES approach is the use of a dither signal that causes persistent exploration of the local decision space, which is needed to estimate gradients for the optimization process. The presence of the dither (e.g. probing signal) implies convergence of the ES scheme to a neighborhood of the optimizer, not the exact value. Though not necessarily a drawback in all applications, when controlling active and reactive power contributions of DER, the probing signal injected in active and reactive power channels may have an opportunity cost as they may prevent the device from maximizing its economic value under certain compensation schemes (by using inverter capacity to probe in the reactive power channel instead of using that capacity for real power injection). Furthermore, the oscillating probes contribute to system losses and may cause other undesirable harmonic content.

The work considered herein significantly alleviates these concerns through introducing an exponentially decaying probe when the individual DER have optimized their portion of the objective function. In so doing, each ES controller will converge to a final value that is closer to the true optimizer and within the neighborhood of attraction when the probing signal is active.

Alternative dither signals (or even the removal of the dither!) in ES have been considered in the past [11], [12], but these techniques do not consider decaying dither when multiple ES controllers are operating in parallel. In a closely related work, the authors of [13] introduce a Lyapunov-based switching criteria to begin the probing signal decay process. However, this switching criteria utilizes knowledge of the underlying system to calculate the Lyapunov function value that governs the decision to switch to decay mode. In the context of controlling DER, this underlying system knowledge translates into system modeling information which may not be available.

In order to enable multiple ES controllers, each managing a separate DER, to have decaying dither signals (that does not utilize any exogenous information about the distribution grid), we introduce an equilibrium-based switching criterion that governs when the probes of the 2D-ES controllers begin to exponentially decay. The specific case of optimization we consider in this work is using DER to enable substation active and reactive power target tracking (similar to the objective considered in [9]). In this paradigm, all of the controllable DER in a given feeder will work together to enable the feeder point of common coupling to have a controllable active and reactive power values. In so doing, we have put in place a framework allowing the feeder to act as an aggregate resource - a step closer to participating in electricity markets or regulation services. In the experiment considered herein, we seek to understand the ability of the 2D-ES controllers to track active and reactive power setpoints, which may change at fixed intervals, with a decaying dithers. Upon setpoint change, the dither signal is re-activated, allowing the controllers to successfully track the new target.

We begin with an overview of the 2D-ES control scheme and a derivation of the equilibrium-based switching criteria, followed by a simulation experiment demonstrating the behavior of the approach. We then 
provide concluding remarks.

\section{Analysis}

This section begins with a discussion of the power flow model used in subsequent analysis as well as a derivation of the objective function for the chosen control activity. This is followed by an overview of the 2D-ES control scheme with equilibrium-based switching criteria that governs the decay of the probing amplitude. The last subsection outlines how setpoints determined by the 2D-ES scheme are rectified to satisfy DER real power, reactive power, and capacity constraints.

\subsection{Objective Function Construction}

Let $G=(\mathcal{N}, \mathcal{L})$ represent a balanced radial distribution system being served by a transmission system link. Here, $\mathcal{N}$ is the set of nodes of the feeder, where $\mathcal{N} \triangleq\{\infty, 0,1, \ldots, n\}$. In this notation, node 0 refers to the distribution system substation, and node $\infty$ represents the transmission system, which is treated as an infinite bus (i.e. we assume control actions taken by the DER do not affect voltage at node $\infty$ ).

We utilize the DistFlow equations [14] to represent the relationship between real and reactive power flows and squared voltage magnitudes between adjacent nodes in $\mathcal{G}$ (Eqs. (1) - (4)).

$$
\begin{aligned}
P_{i} & =p_{i}+u_{i}+\sum_{j:(i, j) \in \mathcal{L}}\left(P_{j}+r_{i j} l_{i j}\right) \\
Q_{i} & =q_{i}+v_{i}+\sum_{j:(i, j) \in \mathcal{L}}\left(Q_{j}+x_{i j} l_{i j}\right) \\
y_{i} & =y_{j}+2 r_{i j} P_{j}+2 x_{i j} Q_{j}+\left(r_{i j}^{2}+x_{i j}^{2}\right) l_{i j} \\
l_{i j} & =\frac{P_{j}^{2}+Q_{j}^{2}}{y_{j}}, \quad \forall(i, j) \in \mathcal{N} \backslash \infty .
\end{aligned}
$$

In our notation, $P_{i}$ and $Q_{i}$ represent the active and reactive power flowing into node $i, p_{i}$ and $q_{i}$ represent node $i$ active and reactive power demand (i.e. the load), and $u_{i}$ and $v_{i}$ denote the active and reactive powers that can be injected/consumed by DER at node $i$. Additionally, losses in that occur over line segment $(i, j)$ are represented by $l_{i j}$, and $y_{i}$ represents the squared voltage magnitude at node $i$. Finally, $r_{i j}$ and $x_{i j}$ represent line segment $(i, j)$ resistance and reactance.

Using the model outlined in Eqs (1)-(4), we formulate the following Optimal Power Flow problem to track real and reactive power targets at the distribution substation while performing feeder voltage regulation:

$$
\begin{array}{cl}
\underset{\boldsymbol{u}, \boldsymbol{v}, y_{i}, P_{i}, Q_{i}}{\operatorname{minimize}} & \alpha_{P}\left(P_{0}-P_{t}\right)^{2}+\alpha_{Q}\left(Q_{0}-Q_{t}\right)^{2} \\
\text { subject to: } & (1)-(4), \\
& \underline{y}_{i} \leq y_{i} \leq \bar{y}_{i}, \quad i \in \mathcal{B}, \\
& \underline{P}_{i} \leq P_{i} \leq \bar{P}_{i}, \quad i \in \mathcal{L}, \\
& \underline{Q}_{i} \leq Q_{i} \leq \bar{Q}_{i}, \quad i \in \mathcal{L}, \\
& \underline{S}_{i} \leq \sqrt{P_{i}^{2}+Q_{i}^{2}} \leq \bar{S}_{i}, \quad i \in \mathcal{L}, \\
& \underline{u}_{i} \leq u_{i} \leq \bar{u}_{i}, \quad i \in \mathcal{N}, \\
& \underline{v}_{i} \leq v_{i} \leq \bar{v}_{i}, \quad i \in \mathcal{N}, \\
& \underline{w}_{i} \leq \sqrt{u_{i}^{2}+v_{i}^{2}} \leq \bar{w}_{i}, \quad i \in \mathcal{N},
\end{array}
$$

where $P_{t}$ and $Q_{t}$ are the substation real and reactive power targets, $\alpha_{P}, \alpha_{Q}$ are scaling factors, and $\mathcal{B} \subset \mathcal{N}$. Here, $\boldsymbol{u}=\left[u_{0}, u_{1}, \ldots, u_{n}\right]^{T}$ and $\boldsymbol{v}=\left[v_{0}, v_{1}, \ldots, v_{n}\right]^{T}$. Without loss of generality, we have assumed the existence of a four quadrant-capable DER at every node in $\mathcal{N} \backslash \infty$.

Let the individual constraints in (5) be represented by the vector $\boldsymbol{c}(\boldsymbol{y}, \boldsymbol{P}, \boldsymbol{Q}, \boldsymbol{u}, \boldsymbol{v}) \in \mathcal{R}^{b \times 1}$ (i.e. we have $b$ inequality constraints). In order to utilize 2D-ES control, we transform the problem (5) into an equivalent form via making successive substitutions of the equality constraints into the inequality constraints and the objective function, and, subsequently, approximating the inequality constraints with penalty functions in the objective, resulting in:

$$
\underset{\boldsymbol{u}, \boldsymbol{v}}{\operatorname{minimize}} J(\boldsymbol{u}, \boldsymbol{v}),
$$

where

$$
\begin{aligned}
J(\boldsymbol{u}, \boldsymbol{v}) & =\alpha_{P}\left(P_{0}(\boldsymbol{u}, \boldsymbol{v})-P_{t}\right)^{2} \\
& +\alpha_{Q}\left(Q_{0}(\boldsymbol{u}, \boldsymbol{v})-Q_{t}\right)^{2}+\lambda_{k} \sum_{k=1}^{b} g\left(c_{k}(\boldsymbol{u}, \boldsymbol{v})\right)
\end{aligned}
$$

and $g\left(c_{k}(\boldsymbol{u}, \boldsymbol{v})\right)=\max \left(0, c_{k}(\boldsymbol{u}, \boldsymbol{v})\right)^{2}$, with $\lambda_{k}$ positive. We note that the local convexity of (7) was established in [9].

\subsection{Control Design}

In this section, we present the 2D-ES scheme with equilibrium-based switching and decaying probing 


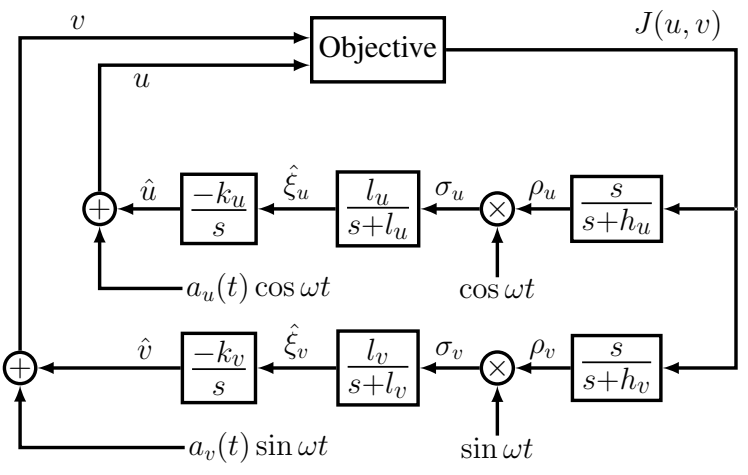

Figure 1. 2D-ES control loop block diagram for a single controller.

amplitudes. The derivation of the equilibrium-based switching criterion can be found in the appendix. An overview of the 2D-ES approach is shown in Figure 1. The goal of the ES controller is to minimize a general convex objective that is, in this case, a function of two independent variables $u$ and $v$. In the Figure, the "Objective" block represents the mapping of all real and reactive power injections into the distribution grid power flows and squared voltage magnitudes (see (1) - (4)) that then are used to construct the objective function (7). The inputs to the "Objective" block consist of sinusoidal perturbations of $a_{u} \cos \omega t$, and $a_{v} \sin \omega t$, added to $\hat{u}$, and $\hat{v}$, respectively, as in (8):

$$
\begin{aligned}
& u(t)=\hat{u}(t)+a_{u}(t) \cos (\omega t) \\
& v(t)=\hat{v}(t)+a_{v}(t) \sin (\omega t)
\end{aligned}
$$

These oscillations propagate throughout the network, resulting in an objective function $J(u, v)$ that is comprised of both DC and oscillatory components. $J(u, v)$ is then split into two channels that feed loops governing the active and reactive power contributions of this DER. In each loop, the DC term is removed by with a highpass filter and the subsequent signal is multiplied by $\cos \omega t$ (in the $u$ channel) and by $\sin \omega t$ (in the $v$ channel). Each signal is then passed through a lowpass filter followed by an integrator before entering a summation junction used to reconstruct the inputs to the "Objective" block. In this scheme, the signals $\hat{\xi}_{u}$ and $\hat{\xi}_{v}$ are estimates of the gradient of $J(u, v)$ with respect to $u$ and $v$.

We consider $m$ individual 2D-ES controllers, each situated at a different node in $\mathcal{G}$, that are operating in parallel, as depicted in Figure 2. We assume that parameters for each of the 2D-ES controllers are all positive and the probing frequencies cannot add or subtract to equal another frequency, or $\omega_{i} \neq \omega_{j}$ and $\omega_{i}+\omega_{j} \neq \omega_{k}$ for distinct $i, j$, and $k$. For simplicity, we

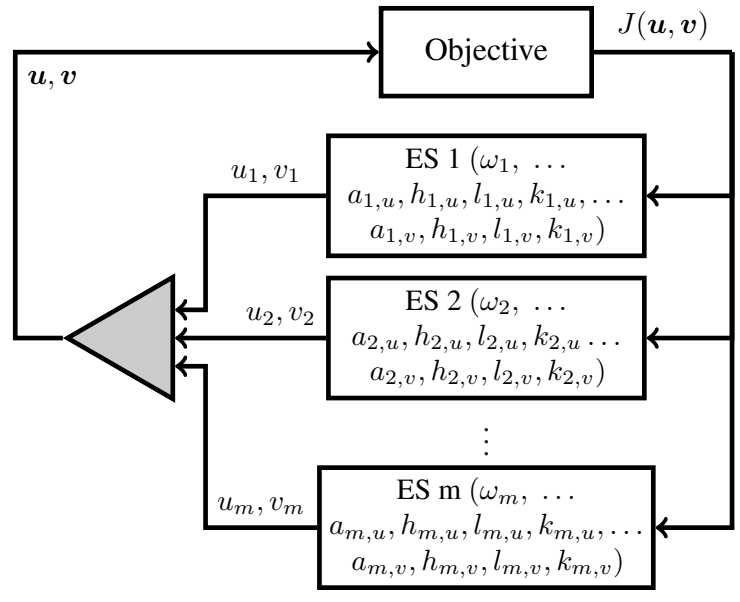

Figure 2. Parallel operation of multiple ES controllers.

assume uniform probing amplitudes and high and low pass filter parameters (see [9] for further explanation) for all controllers (though this assumption does not affect the stability of the approach).

At this stage, we separate the behavior of the 2D-ES scheme into two modes of operation. "Normal mode" corresponds to periods when the ES probe amplitude is set at the simulation initial value, $a_{i, u}^{0}$ or $a_{i, v}^{0}$, and "decay mode" corresponds to periods when the probe amplitude decays starting at $t_{i, u, s w}$ or $t_{i, v, s w}$, where the subscript $s w$ indicates the simulation time when the switching action occurs.

We temporarily omit the subscripts $u$ and $v$ and focus on a single loop in the 2D-ES scheme as the switching criteria logic is identical for both the real and reactive power loops, save the choice of switching thresholds. Let the binary variable $\psi_{i}$ represent the state of the $i^{\text {th }}$ 2D-ES controller, where $\psi_{i}=0$ corresponds to "normal mode" and $\psi_{i}=1$ corresponds to a decaying dither signal (i.e. "decay mode"). Furthermore, let $\bar{J}_{i}$ represent the lowest average objective function value recorded since the transition to $\psi_{i}=1$ :

$$
\bar{J}_{i}=\min \left\{J_{i, a v}(\tau)\right\}, \quad \forall \tau \in\left[t_{i, s w}, t\right] .
$$

Here "av" refers to: $\frac{1}{T_{i}} \int_{t-T_{i}}^{t}(\cdot) d \tau$ and $T_{i}=\frac{\omega_{i}}{2 \pi}$. We define the logic governing switching from "normal mode" to "decay mode" (and vice versa) as:

$$
\psi_{i}= \begin{cases}0 & \text { if }\left|\dot{e}_{i, a v}\right| \geq \bar{e}_{i} \quad \text { or } \quad J_{i, a v} \geq \bar{J}_{i}+\varepsilon_{i} \\ 1 & \text { if }\left|\hat{\xi}_{i, a v}\right| \leq \overline{\hat{\xi}}_{i} \quad \text { and } \quad\left|\sigma_{i, a v}\right| \leq \bar{\sigma}_{i}\end{cases}
$$




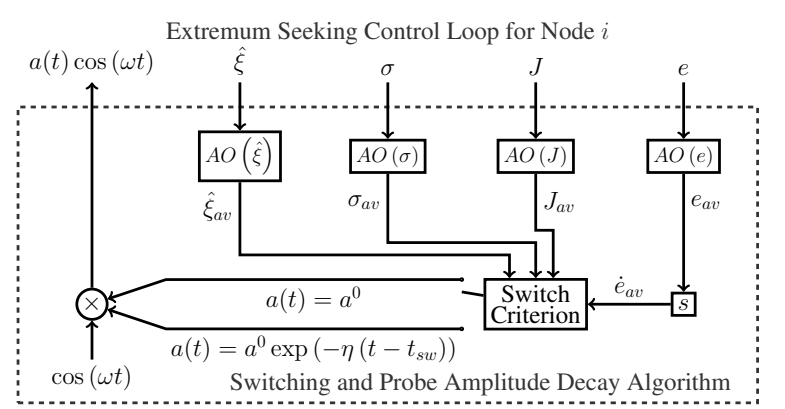

Figure 3. Block diagram of switched 2D-ES system with probe amplitude decay. The averaging operator (AO) refers to $\frac{1}{T_{i}} \int_{t-T_{i}}^{t}(\cdot) d \tau$ and $T_{i}=\frac{\omega_{i}}{2 \pi}$.

where $\bar{e}_{i}, \varepsilon_{i}, \overline{\hat{\xi}}_{i}$, and $\bar{\sigma}_{i}$ are small and positive. The variable $e_{i, a v}$ is the averaged low-pass filtered version of the objective function $J$ (i.e. $e_{i, a v}=J_{i, a v}-\rho_{i, a v}$ ).

The switching logic of (10) will trigger a state change of the ES controller into "decay mode" when the controller enters into a small neighborhood around its equilibrium point. A derivation of the equilibrium point and the switching criterion can be found in the appendix. The controller will exit "decay mode" when the derivative of the averaged objective function exceeds a threshold, or if the objective function value increases away from $\bar{J}_{i}$. The probe amplitude $a_{i}(t)$ in either state of $\psi_{i}$ is given by (11), where $a_{i}^{0}$ is the $i^{t h}$ probe initial value.

$$
a_{i}(t)= \begin{cases}a_{i}^{0} & \text { if } \psi_{i}=0 \\ a_{i}^{0} e^{\left(-\eta_{i}\left(t-t_{i, s w}\right)\right)} & \text { if } \psi_{i}=1\end{cases}
$$

It should be noted that the real and reactive power probes may have different switching parameters, nominal probe amplitude, probe decay rate, and probe decay start time $t_{i, s w}$. Following the change from $\psi_{i}=0$ to $\psi_{i}=1$, the probing amplitude of the $i^{t h}$ ES controller will asymptotically approach 0 , thereby allowing the controller output to reach a value closer to the true optima.

The equilibrium-based switching process is illustrated in Figure 3 that shows the probe amplitude and ES operation mode switching logic for the cosine loop in the 2D-ES scheme (this may be the active or reactive power loop depending on the specific implementation). The switching logic is identical, save for the sine loop after replacing $\cos (\omega t)$ with $\sin (\omega t)$. For clarity of presentation, the subscripts denoting node, and power loop are omitted.

\subsection{DER Constraints and Control Rectification}

In order to ensure the active and reactive power setpoints produced by the 2D-ES control algorithm were feasible with respect to DER real power, reactive power, and apparent power capacity constraints, the infeasible setpoints were rectified via a process outlined in Algorithm 1.

Here, following the notation of Figure $1, \hat{u}$ and $\hat{v}$ refer to the active and reactive power setpoints generated by the 2D-ES controller (where $\hat{w}=\hat{u}+j \hat{v}$ ). The rectification algorithm can be viewed as a projection of any infeasible point onto the constraint set defined in (5). We note that additional constraints (such as DER ramp rates for storage) can easily be incorporated into this framework.

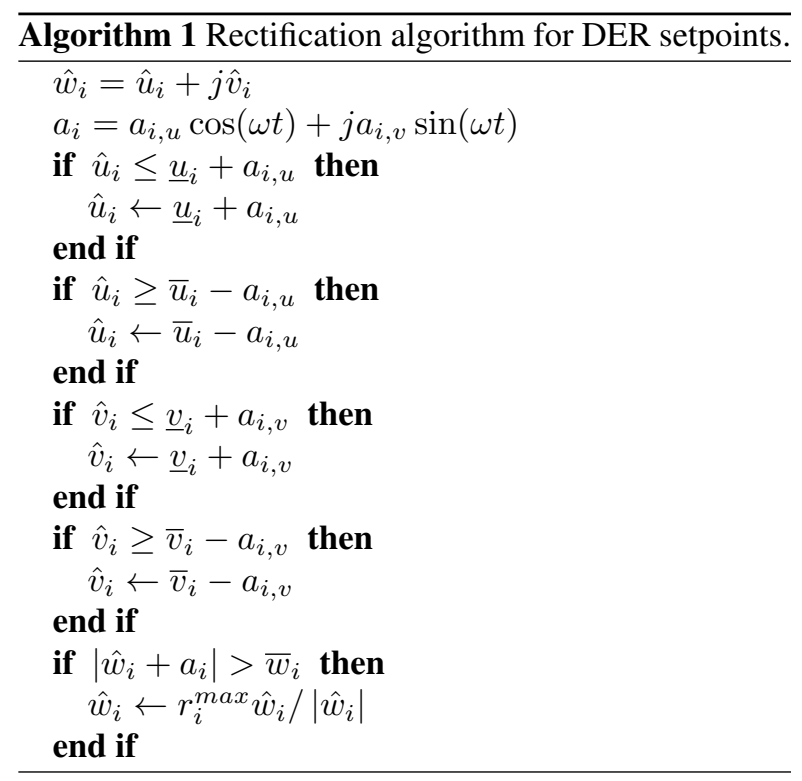

In the last if statement of Algorithm 1, $r_{i}^{\max }$ is the maximum of:

$$
\left|r_{i} \cos \theta+j r_{i} \sin \theta+a_{i, u} \cos \delta+j a_{i, v} \sin \delta\right| \leq \bar{w}_{i},
$$

over $\delta \in[0,2 \pi]$ where $\theta=\arctan \left(\hat{v}_{i} / \hat{u}_{i}\right)$.

The rectification process is illustrated in Figure 4 and Figure 5 that show how setpoints computed by the ES controller are adjusted so that the DER output always remains feasible. In Figure 4, infeasible setpoints are represented by red dots and the rectified feasible setpoints are shown as blue dots. The ellipses represent the effect of the probing (dither) signal, that causes a perturbation around the present setpoint. In Figure 5 , dashed lines represent the boundary of the feasible setpoints for two cases of probe amplitudes, and ellipses represent the corresponding probes. Note that after 


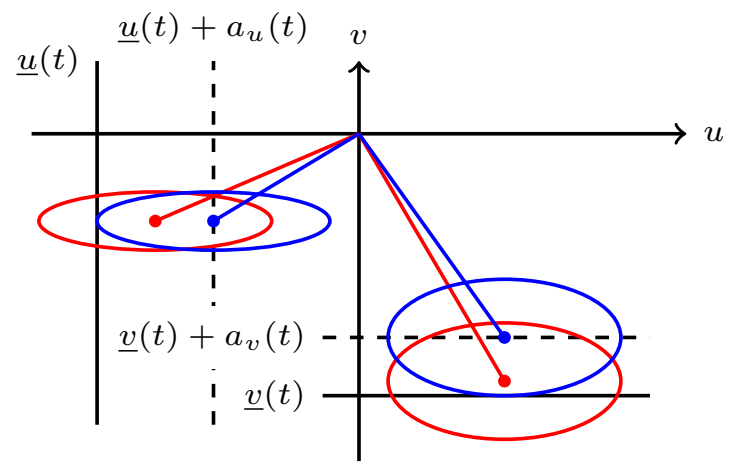

Figure 4. Rectification for minimum real power constraint and minimum reactive power constraint. Infeasible setpoints are colored red, and rectified setpoints are blue. The corresponding probes are the ellipses centered at each setpoint.

rectification, the setpoint value plus the effect of the dither always remains feasible. This ensures that the ES controllers will always be able to extract the gradient of the objective function. In the event of probing amplitude decay, it will be necessary to continually re-evaluate Algorithm 1.

\section{Simulations}

To investigate the behavior of ES control with decaying probe amplitude, simulations were conducted in which multiple DER, each controlled by a 2D-ES algorithm, dispatch their active and reactive power contributions to track both real power and reactive power targets at the feeder head. This particular objective function is represented by:

$$
\begin{aligned}
J(\mathbf{u}, \mathbf{v})= & 100\left(P_{0}(\mathbf{u}, \mathbf{v})-P_{t}\right)^{2} \ldots \\
& +100\left(Q_{0}(\mathbf{u}, \mathbf{v})-Q_{t}\right)^{2}
\end{aligned}
$$

where coefficients of 100 were chosen to scale the objective function output to a value on the order of 1 .

Simulations were conducted on a modified version of the IEEE 37 node test feeder (balanced), the topology of which can be seen in Figure 6. The voltage regulator between nodes 799 and 701 was omitted, and the switch between 709 and 775 was replaced by a line with configuration 724 and length of $50 \mathrm{ft}$. The network was balanced, such that all entities on phases $b$ and $c$ were eliminated, as were cross phase impedances. Network entities on phase $a$ were retained. All demands were multiplied by a factor of 1.5. Loads were assumed to follow a ZIP model of the form:

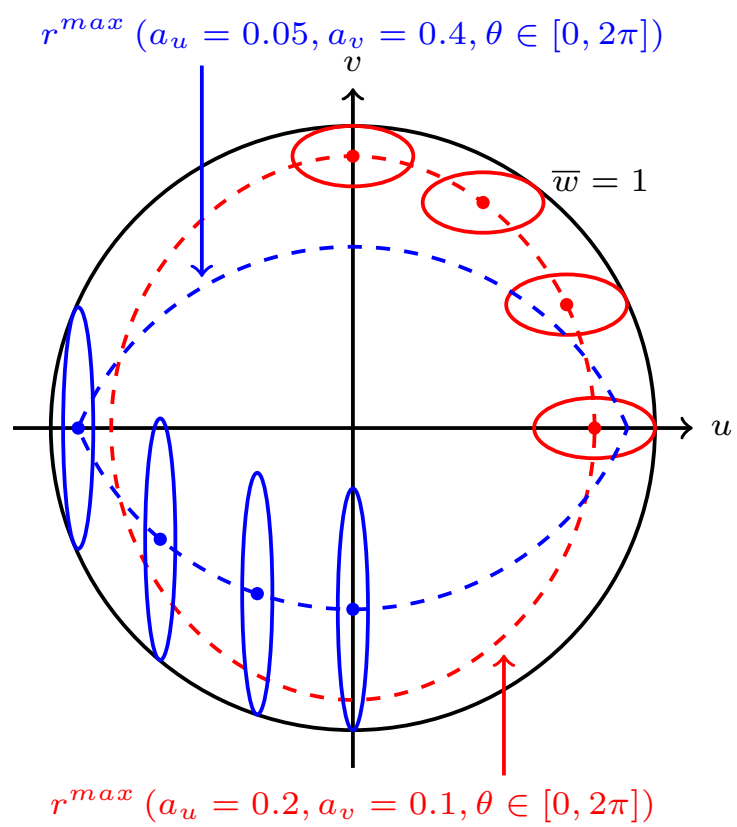

Figure 5. Illustration of the effective rectification for inverter apparent power constraint. Maximum allowable setpoint for two cases of probe amplitudes represented by the dashed lines. The ellipses represent the corresponding probes, showing the entirety of the probe remaining within the feasible region.

$$
\begin{aligned}
& p_{i}\left(y_{i}\right)=p_{i}\left(\beta_{i, S}+\beta_{i, I} y_{i}^{\frac{1}{2}}+\beta_{i, Z} y_{i}\right) \\
& q_{i}\left(y_{i}\right)=q_{i}\left(\beta_{i, S}+\beta_{i, I} y_{i}^{\frac{1}{2}}+\beta_{i, Z} y_{i}\right)
\end{aligned}
$$

with ZIP model parameters: $\beta_{i, S}=0.75, \beta_{i, I}=$ $0.10, \beta_{i, Z}=0.15 \forall i \in \mathcal{N}$

DER were placed at nodes $\mathcal{C}=$ $\{702,725,729,731,735,711\}$, and were assumed to be capable of four-quadrant operation. Each DER had its own 2D-ES controller allowing for simultaneous management of real and reactive power. The real and reactive control loops for each 2D-ES controller used the same probing frequency $f$, as defined in Table 1. All controller parameters, including high pass filter frequencies, low-pass filter frequencies, integrator gains, and initial probe amplitude can be found in Table 1. The same values for each parameter were assigned to both the real and reactive power control loops, and therefore we have omitted the subscripts of $u$ and $v$ in Table 1. The dither decay rate, and switching criteria values are also given in Table 1. 
Table 1. Extremum seeking controller and operation mode switch parameters. We omit the subscripts $u$ and $v$ as we assign the same parameters to the real and reactive power control loops.

\begin{tabular}{|l|c|c|c|c|c|c|c|c|c|c|}
\hline Node & $f_{i}[\mathrm{~Hz}]$ & $h_{i}[\mathrm{~Hz}]$ & $l_{i}[\mathrm{~Hz}]$ & $k_{i}$ & $a_{i}^{0}$ & $\eta_{i}$ & $\overline{\hat{\xi}}_{i, a v}$ & $\bar{\sigma}_{i, a v}$ & $\bar{e}_{i, a v}$ & $\varepsilon_{i}$ \\
\hline 702 & 1.00 & 1.0 & 1.0 & 0.05 & 0.005 & 0.1 & 0.01 & 0.2 & 0.5 & 0.2 \\
725 & $\sqrt{2}$ & 1.0 & 1.0 & 0.05 & 0.005 & 0.1 & 0.01 & 0.2 & 0.5 & 0.2 \\
729 & $\sqrt{3}$ & 1.0 & 1.0 & 0.05 & 0.005 & 0.1 & 0.01 & 0.2 & 0.5 & 0.2 \\
731 & $\sqrt{5}$ & 1.0 & 1.0 & 0.05 & 0.005 & 0.1 & 0.01 & 0.2 & 0.5 & 0.2 \\
735 & $\sqrt{7}$ & 1.0 & 1.0 & 0.05 & 0.005 & 0.1 & 0.01 & 0.2 & 0.5 & 0.2 \\
711 & $\sqrt{11}$ & 1.0 & 1.0 & 0.05 & 0.005 & 0.1 & 0.01 & 0.2 & 0.5 & 0.2 \\
\hline
\end{tabular}

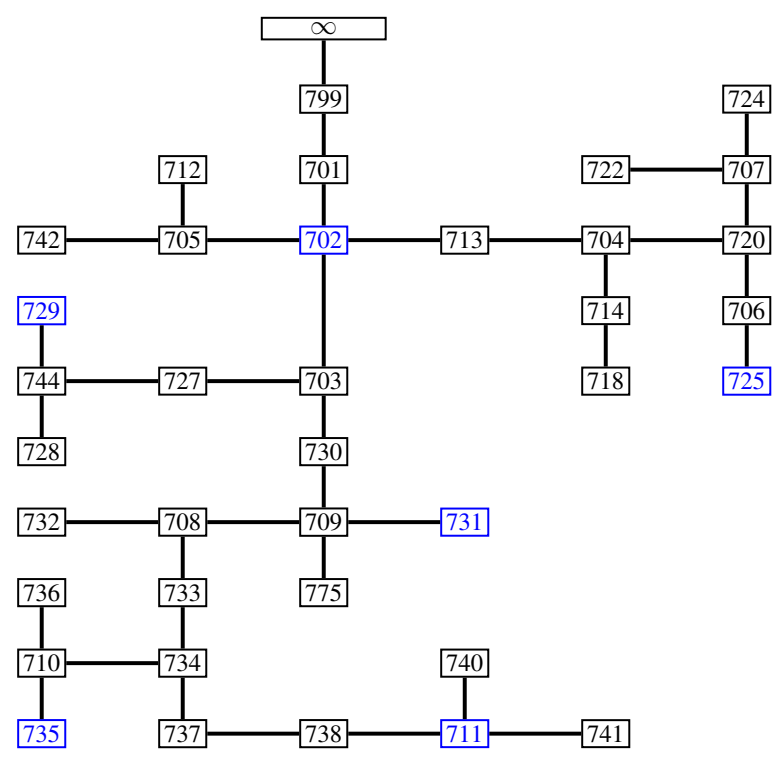

Figure 6. IEEE 37 node test feeder topology. Nodes with DER are blue.

In the simulations, the real power target, $P_{t}$ was set to 0.35 p.u. for $0 \leq t \leq 30$, then 0.25 p.u. for $30 \leq$ $t \leq 60$, then ramps linearly in time from 0.25 to 0.35 for $60 \leq t \leq 90$, then remains constant at 0.35 from $90 \leq t \leq 120$. The reactive power target, $Q_{t}$ was set to 0.15 p.u. for $0 \leq t \leq 30$, then 0.05 p.u. for $30 \leq$ $t \leq 60$, then ramps linearly in time from 0.05 to 0.15 for $60 \leq t \leq 90$, then remains constant at 0.15 from $90 \leq t \leq 120$.

Figure 7 plots the feeder head real and reactive power, and and the associated substation power targets. The 2D-ES controllers successfully converge to their respective targets when the targets are static, and follow the ramp function, albeit with some lag.

Figure 8 shows the value of (13) and the low-pass filtered objective function for node 735. As is shown in the Figure, the 2D-ES controllers collectively minimize the global objective function when tracking both sets of targets.

Figure 9 shows with greater detail the substation real

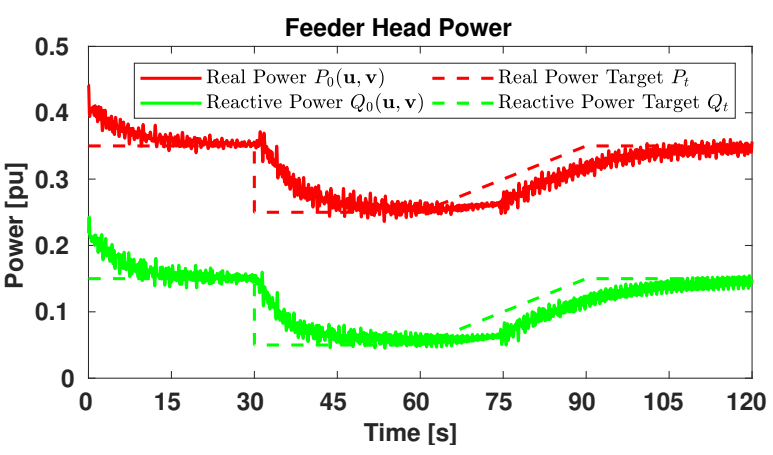

Figure 7. Feeder head power and power references values.

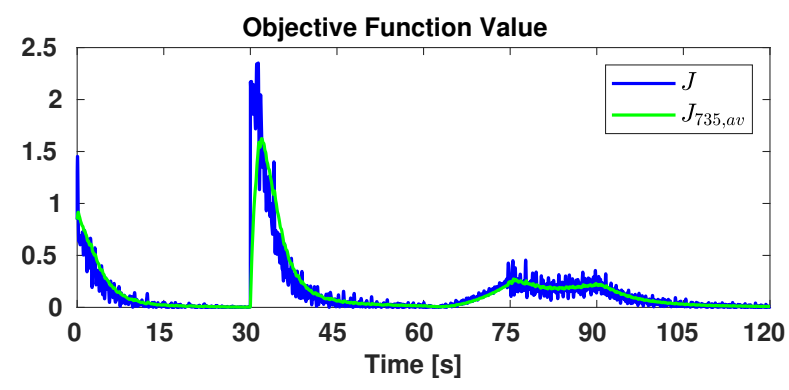

Figure 8. Objective function, in blue, and low-pass filtered objective function for node 735 , in green. The low-pass filtered objective function is the same for all controllers at all nodes.

and reactive power during and after the linear increase in targets from $60 \leq t \leq 90$, and the corresponding effect on the objective function and switching threshold for node 735. The objective function initially increases as the targets increase without the the substation real and reactive power being able to keep up. After the low-pass filtered objective function crosses the threshold of $\bar{J}_{735}+\varepsilon_{735}$, all probes are switched into "normal mode", at which point the probes are able to follow the changing target values and minimize the objective function.

Figs. 10 and 11, show the active and reactive power contributions, as well as the probe amplitudes of the 

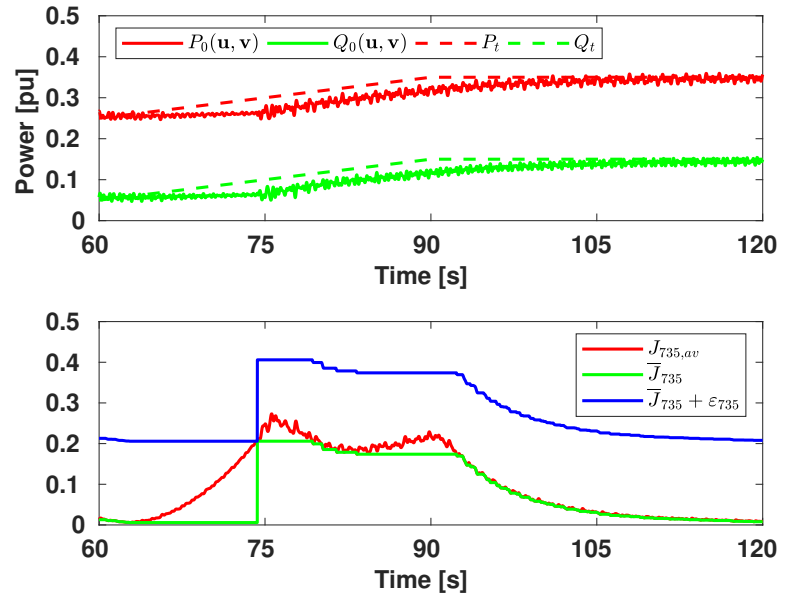

Figure 9. Detail of the ramp in power reference values and switching criterion. The top subplot shows the monotonic increase in reference values between simulation time of $\mathbf{6 0}$ and $\mathbf{9 0}$ seconds. The lower subplot shows the the low-pass filtered objective function for node 735 and the switching threshold values.

real and reactive power probes $\left(a_{i, u}\right.$ and $\left.a_{i, v}\right)$, for DER operating at node 702 , and 735 , respectively. It is evident that the real and reactive power probes enter "decay" mode at different times, for the controllers at nodes 702 and 735 .

\section{Discussion and Conclusion}

This paper considered the use of 2D-ES control to manage DER active and reactive power contributions with the intention of enabling substation power target tracking. We augmented our previously designed control scheme (presented in [9]) with an equilibrium-based switching criterion to enable the individual DER dither signals to exponentially decay once the controllers had achieved their optimum values. Simulation results show the equilibrium-based switching criterion allows the 2D-ES controllers to track time-varying substation active and reactive power targets and with decaying dither signals. As such, the ES controllers converge to points that are substantially closer to the true optimum value (e.g. the targets) than when the dither signals were active. The approach remains completely model free (i.e. no knowledge of the system and global load information is required). Although not presented here for space considerations, we have conducted several experiments varying the ZIP composition for active and reactive power loads in the simulation. Including cases where the constant power portion of the active and reactive loads differed
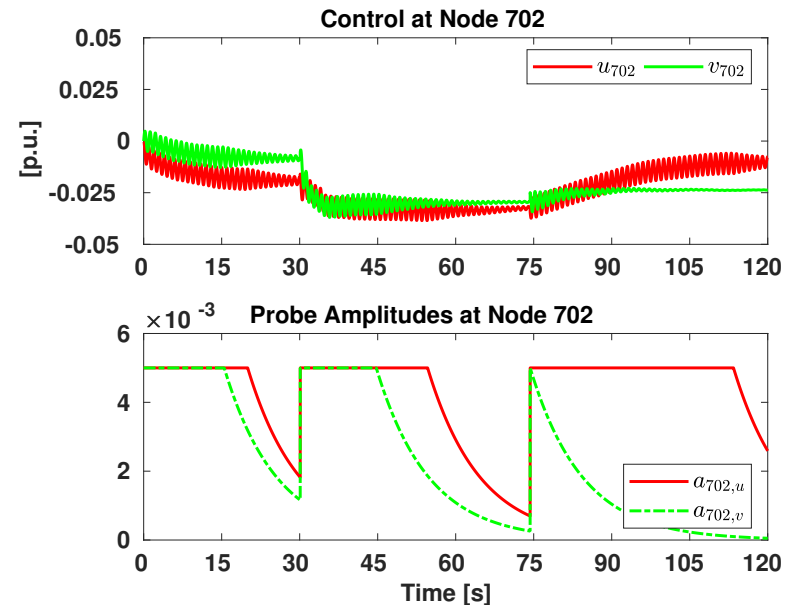

Figure 10. Real and reactive power control, and probe amplitudes, at node 702 .
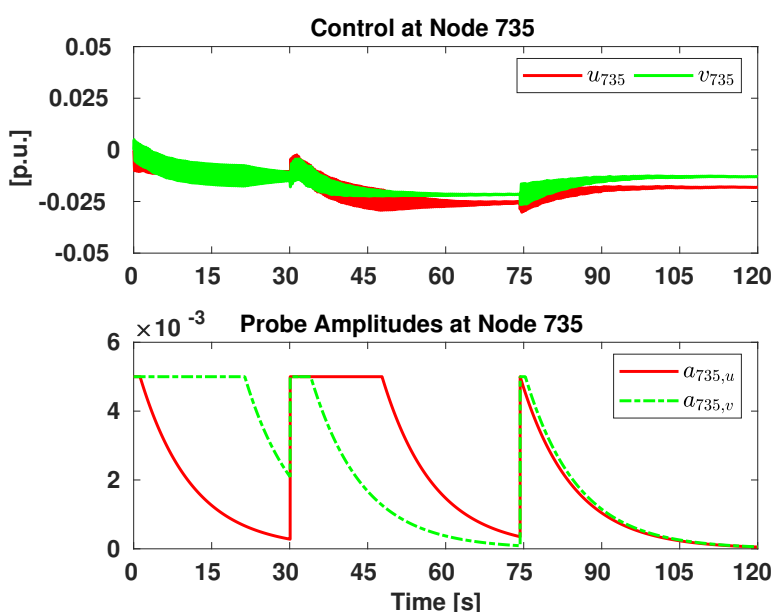

Figure 11. Real and reactive power control, and probe amplitudes, at node 735 .

greatly, the ES algorithm with the decaying dither feature successfully tracked a time-varying objective function.

It is possible that the presence of the dither signal could adversely affect the distribution system. The frequency of the individual probes is largely a design choice. The ES algorithm (in both theory and practice) has been shown to converge for a variety of different frequency choices. There are some parts of the signal spectrum where it would be undesirable to add energy (to avoid flicker, for example). However, there are other parts of the spectrum that could be relatively noise free that, were probing to occur at these frequencies, would not cause adverse system effects. Our planned future work will focus on hardware field demonstrations with utility partners to identify which parts of the signal spectrum are most ideal for sinusoidal probing. It is 
worth noting that the main contribution of this work will mitigate exactly this issue. By encouraging the dither signals to decay when the DER have reached their respective optima, we significantly reduce the duration of adverse system impacts (if these even occur).

As the analysis for the original 2D-ES scheme shows, the proposed extended 2D-ES can be used to optimize a wide range of objectives in addition to target tracking [9]. In the future we plan to conduct larger numerical experiments of unbalanced distribution systems, as well as provide analytically-derived criteria that show the relationship between probing decay rate and the choice of other parameters in the 2D-ES loops.

\section{References}

[1] I. Penn, "California will require solar power for new homes," New York Times, May 2018.

[2] E. Dall'Anese, H. Zhu, and G. Giannakis, "Distributed optimal power flow for smart microgrids," IEEE Trans. Smart Grid, vol. 4, no. 3, pp. 1464-1475, 2013.

[3] L. Gan, N. Li, U. Topcu, and S. H. Low, "Exact convex relaxation of optimal power flow in radial networks," IEEE Transactions on Automatic Control, vol. 60, no. 1, pp. 72-87, 2015.

[4] E. DallAnese, K. Baker, and T. Summers, "Chance-constrained ac optimal power flow for distribution systems with renewables," IEEE Trans. Power Syst., vol. 32, pp. 3427-3438, Sept 2017.

[5] Y. Zhang, M. Hong, E. Dall'Anese, S. Dhople, and $\mathrm{Z}$. Xu, "Distributed controllers seeking ac optimal power flow solutions using admm," IEEE Trans. Smart Grid, vol. pp, pp. 1-13, 2017.

[6] M. D. Sankur, R. Dobbe, A. von Meier, E. M. Stewart, and D. B. Arnold, "Optimal voltage phasor regulation for switching actions in unbalanced distribution systems," arXiv preprint arXiv:1804.02080, 2018.

[7] M. Krsti and H. Wang, "Stability of extremum seeking feedback for general nonlinear dynamic systems," Automatica, vol. 36, no. 4, pp. 595 - 601, 2000.

[8] K. B. Ariyur and M. Krstić, Real-time optimization by extremum-seeking control. John Wiley \& Sons, 2003.

[9] D. B. Arnold, M. D. Sankur, M. Negrete-Pincetic, and D. Callaway, "Model-free optimal coordination of distributed energy resources for provisioning transmission-level services," IEEE Trans. Power Syst., vol. 33, pp. 817-829, Jan. 2018.

[10] J. Johnson, S. Gonzalez, and D. B. Arnold, "Experimental distribution circuit voltage regulation using der power factor, volt-var, and extremum seeking control methods," in Photovoltaic Specialists Conference, IEEE, 2017.

[11] B. G. B. Hunnekens, M. A. M. Haring, N. van de Wouw, and H. Nijmeijer, "A dither-free extremum-seeking control approach using 1st-order least-squares fits for gradient estimation," in Conference on Decision and Control, pp. 2679-2684, Dec 2014.

[12] D. DeHaan and M. Guay, "Extremum-seeking control of state-constrained nonlinear systems," Automatica, vol. 41, no. 9 , pp. $1567-1574,2005$.
[13] S. J. Moura and Y. A. Chang, "Lyapunov-based switched extremum seeking for photovoltaic power maximization," Control Engineering Practice, vol. 21, no. 7, pp. $971-980,2013$.

[14] M. E. Baran and F. F. Wu, "Network reconfiguration in distribution systems for loss reduction and load balancing," IEEE Trans. Power Del., vol. 4, no. 2, pp. 1401-1407, 1989.

\section{Appendix}

Consider a convex objective function $J(\boldsymbol{u}, \boldsymbol{v})$. Now, define $\hat{\boldsymbol{u}} \in \mathbb{R}^{m \times 1}$ and $\hat{\boldsymbol{v}} \in \mathbb{R}^{m \times 1}$ as the vectors consisting of the signals after the integrators for each 2D-ES controller in the channels $\boldsymbol{u}$ and $\boldsymbol{v}$, respectively. Furthermore, let $\boldsymbol{q}(t)=\left[\cos \left(\omega_{1} t\right), \ldots, \cos \left(\omega_{m} t\right)\right]^{T}$ represent a vector consisting of all cosine perturbation signals and $\boldsymbol{r}(t)=\left[\sin \left(\omega_{1} t\right), \ldots, \sin \left(\omega_{m} t\right)\right]^{T}$ represent a vector consisting of all sine perturbation signals. The vector of control inputs can then be expressed as: $\boldsymbol{u}=$ $\hat{\boldsymbol{u}}+a_{u} \boldsymbol{q}(t)$ and $\boldsymbol{v}=\hat{\boldsymbol{v}}+a_{v} \boldsymbol{r}(t)$, where $a_{u}$ and $a_{v}$ are scalars. The dynamics of the system of controllers outlined in Figs. 1 - 2 can now be expressed in vector form as:

$$
\begin{aligned}
& \dot{e_{u}}=h_{u}\left(J\left(\hat{\boldsymbol{u}}+a_{u} \boldsymbol{q}(t), \hat{\boldsymbol{v}}+a_{v} \boldsymbol{r}(t)\right)-e_{u}\right) \\
& \dot{e_{v}}=h_{v}\left(J\left(\hat{\boldsymbol{u}}+a_{u} \boldsymbol{q}(t), \hat{\boldsymbol{v}}+a_{v} \boldsymbol{r}(t)\right)-e_{v}\right) \\
& \dot{\boldsymbol{\xi}}_{u}=-l \boldsymbol{\xi}_{u}+ \\
& l \boldsymbol{q}(t)\left(J\left(\hat{\boldsymbol{u}}+a_{u} \boldsymbol{q}(t), \hat{\boldsymbol{v}}+a_{v} \boldsymbol{r}(t)\right)-e_{u}\right) \\
& \dot{\boldsymbol{\xi}}_{v}=-l \boldsymbol{\xi}_{v}+ \\
& l \boldsymbol{r}(t)\left(J\left(\hat{\boldsymbol{u}}+a_{u} \boldsymbol{q}(t), \hat{\boldsymbol{v}}+a_{v} \boldsymbol{r}(t)\right)-e_{v}\right) \\
& \dot{\hat{\boldsymbol{u}}}=-k_{u} \boldsymbol{\xi}_{u} \\
& \dot{\hat{\boldsymbol{v}}}=-k_{v} \boldsymbol{\xi}_{v},
\end{aligned}
$$

where $e_{u}$ and $e_{v}$ are scalars representing the DC portion of the objective function and $\boldsymbol{\xi}_{u}, \boldsymbol{\xi}_{v}, \hat{\boldsymbol{u}}, \hat{\boldsymbol{v}} \in \mathbb{R}^{m \times 1}$ are vectors consisting of signals following the low pass filter blocks depicted in Figure 1.

In order to put the system into a form for which averaging is applicable, we adopt a change of timescales from $t$ to $\tau=\omega_{\tau} t$, where $\omega_{\tau}$ is the lowest common multiple of all system probing frequencies such that the entire system is $\tau$-periodic. Following this change of variables, the perturbation frequency of the $i^{t h}$ 2D-ES controller can be written as: $\omega_{i}=\gamma_{i} \omega_{\tau}$, where $\gamma_{i}$ is constant and positive. In the $\tau=\omega t$ timescale, the system of (15) -(20) becomes: 


$$
\begin{aligned}
& \dot{e_{u}}=\frac{h}{\omega_{\tau}}\left(J\left(\hat{\boldsymbol{u}}+a_{u} \boldsymbol{q}(\tau), \hat{\boldsymbol{v}}+a_{v} \boldsymbol{r}(\tau)\right)-e_{u}\right) \\
& \dot{e_{v}}=\frac{h}{\omega_{\tau}}\left(J\left(\hat{\boldsymbol{u}}+a_{u} \boldsymbol{q}(\tau), \hat{\boldsymbol{v}}+a_{v} \boldsymbol{r}(\tau)\right)-e_{v}\right) \\
& \dot{\boldsymbol{\xi}}_{u}=-\frac{l}{\omega_{\tau}} \boldsymbol{\xi}_{u}+ \\
& \frac{l}{\omega_{\tau}} \boldsymbol{q}(\tau)\left(J\left(\hat{\boldsymbol{u}}+a_{u} \boldsymbol{q}(\tau), \hat{\boldsymbol{v}}+a_{v} \boldsymbol{r}(\tau)\right)-e_{u}\right) \\
& \dot{\boldsymbol{\xi}_{v}}=-\frac{l}{\omega_{\tau}} \boldsymbol{\xi}_{v}+ \\
& \frac{l}{\omega_{\tau}} \boldsymbol{r}(\tau)\left(J\left(\hat{\boldsymbol{u}}+a_{u} \boldsymbol{q}(\tau), \hat{\boldsymbol{v}}+a_{v} \boldsymbol{r}(\tau)\right)-e_{v}\right) \\
& \dot{\hat{\boldsymbol{u}}}=-\frac{k_{u}}{\omega_{\tau}} \boldsymbol{\xi}_{u} \\
& \dot{\hat{\boldsymbol{v}}}=-\frac{k_{v}}{\omega_{\tau}} \boldsymbol{\xi}_{v},
\end{aligned}
$$

where the dot notation on the derivative terms on the left hand side of the preceding equations now indicates $\frac{d}{d \tau}$. As $\boldsymbol{q}(t)$ and $\boldsymbol{r}(t)$ are vectors consisting of sinusoids, their inner products with themselves and each other have the following properties:

$$
\begin{aligned}
\boldsymbol{q}(\tau) \boldsymbol{r}^{T}(\tau) & =\frac{1}{2} \boldsymbol{X}_{m \times m}, \\
\boldsymbol{q}(\tau) \boldsymbol{q}^{T}(\tau) & =\frac{1}{2}\left(\boldsymbol{I}_{m \times m}+\boldsymbol{Y}_{m \times m}\right), \\
\boldsymbol{r}(\tau) \boldsymbol{r}^{T}(\tau) & =\frac{1}{2}\left(\boldsymbol{I}_{m \times m}+\boldsymbol{Z}_{m \times m}\right),
\end{aligned}
$$

where

$$
\begin{aligned}
& \boldsymbol{X}(j, k)=\sin \left(\left(\gamma_{j}+\gamma_{k}\right) \omega_{\tau} \tau\right)+\sin \left(\left(\gamma_{j}-\gamma_{k}\right) \omega_{\tau} \tau\right) \\
& \boldsymbol{Y}(j, j)=\cos \left(2 \gamma_{j} \omega_{\tau} \tau\right), \\
& \boldsymbol{Y}(j, k)=\cos \left(\left(\gamma_{j}-\gamma_{k}\right) \omega_{\tau} \tau\right)+\cos \left(\left(\gamma_{j}+\gamma_{k}\right) \omega_{\tau} \tau\right), \\
& \boldsymbol{Z}(j, j)=-\cos \left(2 \gamma_{j} \omega_{\tau} \tau\right), \\
& \boldsymbol{Z}(j, k)=\cos \left(\left(\gamma_{j}-\gamma_{k}\right) t\right)-\cos \left(\left(\gamma_{j}+\gamma_{k}\right) \omega_{\tau} \tau\right)
\end{aligned}
$$

Given these properties, we can apply averaging (21)-(26), and integrate over the period 0 to $\omega_{\tau} / 2 \pi$ :

$$
\begin{gathered}
\dot{e}_{u, a v}=\frac{h}{2 \pi}\left(J_{a v}(\boldsymbol{u}, \boldsymbol{v})-e_{u, a v}\right) \\
\dot{e}_{v, a v}=\frac{h}{2 \pi}\left(J_{a v}^{*}(\boldsymbol{u}, \boldsymbol{v})-e_{v, a v}\right) \\
\dot{\boldsymbol{\xi}}_{u, a v}=\frac{l}{2 \pi}\left(-\boldsymbol{\xi}_{u, a v}+\frac{a_{u}}{2} \nabla_{\boldsymbol{u}} J_{a v}\left(\boldsymbol{u}_{a v}, \boldsymbol{v}_{a v}\right)\right) \\
\dot{\boldsymbol{\xi}}_{v, a v}=\frac{l}{2 \pi}\left(-\boldsymbol{\xi}_{v, a v}+\frac{a_{v}}{2} \nabla_{\boldsymbol{v}} J_{a v}\left(\boldsymbol{u}_{a v}, \boldsymbol{v}_{a v}\right)\right) \\
\dot{\boldsymbol{\boldsymbol { u }}}_{a v}=-\frac{k_{u}}{2 \pi} \boldsymbol{\xi}_{u, a v} \\
\dot{\hat{\boldsymbol{v}}}_{a v}=-\frac{k_{v}}{2 \pi} \boldsymbol{\xi}_{v, a v}
\end{gathered}
$$

where we have utilized a first order Taylor expansion of $J(\boldsymbol{u}, \boldsymbol{v})$ around the point $\left(a_{u} \boldsymbol{q}(t), a_{v} \boldsymbol{r}(t)\right)$. Here "av" refers to: $\frac{1}{T} \int_{0}^{T}(\cdot) d \tau$ and $T=\frac{\omega_{\tau}}{2 \pi}$. Stability of this system is proven in [9].

Noting that (37) - (40) do not depend on $e_{u, a v}$ or $e_{v, a v}$, we continue the analysis of the dynamics of $\boldsymbol{\xi}_{u, a v}, \boldsymbol{\xi}_{v, a v}, \quad \boldsymbol{u}_{a v}$ and $\boldsymbol{v}_{a v}$. The reduced order system has equilibrium when $\left(\boldsymbol{\xi}_{u, a v}, \boldsymbol{\xi}_{v, a v}, \nabla_{\boldsymbol{u}} J_{a v}\left(\boldsymbol{u}_{a v}, \boldsymbol{v}_{a v}\right), \nabla_{\boldsymbol{v}} J_{a v}\left(\boldsymbol{u}_{a v}, \boldsymbol{v}_{a v}\right)\right)$ are all equal to 0 . Conveniently, the components of $\nabla_{\boldsymbol{v}} J_{a v}\left(\boldsymbol{u}_{a v}, \boldsymbol{v}_{a v}\right)$ and $\nabla_{\boldsymbol{v}} J_{a v}\left(\boldsymbol{u}_{a v}, \boldsymbol{v}_{a v}\right)$ are the signals $\sigma_{u}$ and $\sigma_{v}$ following the demodulation operation shown in Figure 1. As such we design the switching criteria for the $i^{t h}$ 2D-ES controller to initiate the decay of $a_{i, u}$ at $t=t_{i, u, s w}$, and $a_{i, v}$ at $t=t_{i, v, s w}$, as:

$$
\begin{aligned}
& u: \quad\left|\xi_{i, u, a v}\right| \leq \bar{\xi}_{i, u, a v} \quad \& \quad\left|\sigma_{i, u, a v}\right| \leq \bar{\sigma}_{i, u, a v} \\
& v: \quad\left|\xi_{i, v, a v}\right| \leq \bar{\xi}_{i, v, a v} \quad \& \quad\left|\sigma_{i, v, a v}\right| \leq \bar{\sigma}_{i, v, a v}
\end{aligned}
$$

